TRAMES, 2009, 13(63/58), 2, 95-108

\title{
FROM PERSON DESCRIPTIONS TO INTERVIEWING METHODS: WHAT CAN BE DONE TO IMPROVE CHILD WITNESSES' TESTIMONIES?
}

\author{
Kristjan Kask ${ }^{1}$ and Ray Bull ${ }^{2}$ \\ ${ }^{1}$ University Nord, Tallinn, and ${ }^{2}$ University of Leicester
}

\begin{abstract}
Recently an increasing number of studies have been conducted in psychology and law. This paper reviews more closely relevant research concerning child witnesses. Several factors which may affect witness testimonies are covered, including theoretical issues regarding person descriptions such as verbal and visual processing, and characteristics influencing these processes. Also, archival and empirical studies of person descriptions and characteristics of witnesses are reviewed. Finally, the outcomes of different methods which were intended to increase the quality and quantity of children's person descriptions (but have had limited success) are overviewed such as the standard model for comparison. In order to achieve the best balance between quantity and quality in children's accounts, it is recommended that interviewers learn to use structured methods such as the NICHD protocol or the cognitive interview.
\end{abstract}

DOI: $10.3176 /$ tr.2009.2.01

Keywords: child witnesses, person descriptions, standard, interviewing techniques, cognitive interview, NICHD protocol

\section{Introduction}

In the past 25 years, more and more research has been conducted on child witnesses. In many countries young children are increasingly being called to testify in criminal cases, particularly in sexual abuse cases (Schepard 2004). Many of the court cases concerning children involve allegations of sexual or physical abuse by members of the child's family or other persons known to the child. However, children may also be victims of or witnesses to crimes committed by strangers, such as traffic accidents, thefts, assaults, and murders. Importantly, however, children are often considered unreliable witnesses. For example, surveys of adults' beliefs about child eyewitnesses have found that children are often 
viewed with scepticism and that adult witnesses are judged as more credible (Pozzulo et al. 2007).

This paper reviews relevant research concerning children's person descriptions. The first part of the paper focuses on adults because with regard to several factors that may affect child witnesses only studies with adults have been conducted. An overview is given of theoretical issues regarding person descriptions such as verbal and visual processing, and characteristics influencing these processes, followed by a review of archival and empirical studies of person descriptions by adults and characteristics of witnesses. After that, studies on children's person descriptions are reviewed. Finally, the outcomes of different methods which were designed to increase the quality and quantity of children's person descriptions are overviewed. The issues of eyewitness identification concerning children are not included into this review due to large number of factors related to this topic. In this paper 'children' is a term used to refer to those below 14 years of age.

\section{Theoretical issues in descriptions}

Eyewitnesses play a crucial role in bringing perpetrators of crime to justice. The police rely very much on witness testimony, especially at the beginning of their investigations (Fisher and Geiselman 1992). Collecting person descriptions from witnesses is also one of the regular information-collecting tools (Meissner et al. 2006).

Person descriptions are usually verbal reproductions of (visually) perceived people (Sporer 1996). Such descriptions generally contain reference to physical appearance (mainly face, height and weight), stature and clothing. Descriptions regarding the face can be crucial for identifying a person. However, our vocabulary for expressing the physical aspects of faces is rather limited, when compared to the large number of adjectives available for describing character traits (Shepherd and Ellis 1996). People tend toward providing character attributes in their descriptions of a once-seen person, even when they have been explicitly instructed to provide only physical descriptions (Sporer 1996).

One explanation of why it is hard to recall physical aspects of faces (rather than character traits) was provided by Paivio $(1969,1971)$ who suggested that verbal information is processed differently from imaginal (i.e. visual) information. However, he also contended that we use both imaginal and verbal codes to some extent for representing either sort of information (dual coding). According to Paivio (1969) (i) for physical stimuli our mental images are analogue codes (a form of knowledge representation that preserves the main perceptual features of whatever is being represented), but (ii) our mental representations for verbal information are represented in a symbolic code (a form of knowledge representation that stands for something and does not perceptually resemble whatever is being represented).

Breznitz (2002), among others, has claimed that the auditory (e.g. verbal) and visual systems process information differently. Discrimination and identification 
are achieved faster through the visual than the auditory route because the visual system processes information holistically whereas the auditory system processes information sequentially. As the visual route is faster and holistic, this would apply to processes like face or person recognition. This also partly explains why we have difficulties in coding visually perceived information into a verbal mode. It also explains why it is difficult to 'translate' visual memories into verbal retrieval.

The visual and verbal encoding and decoding processes involved in an exchange between the witness and the investigator could be as follows (Sporer 1996). First, the witness has to transform the visual aspects of the target into a verbal person description. One of the problems here is that faces are best encoded holistically (Wegner and Ingvalson 2002), whereas a verbal description uses more a piecemeal approach involving the labelling of the individual features (in which the object of description is broken down into singular details) (Sporer 1992a). The police usually require featural rather than holistic descriptors.

Second, the description is usually conveyed verbally by the witness to the investigator who often has to re-transform this description into a visual representation of the described person. One problem here is that a witness may describe a person as having a 'hooked nose', but for somebody else to hear/read this description there is an almost infinite number of curvatures imaginable that would still be compatible with the description 'hooked nose' (but no longer to the nose originally perceived by the witness). Or if the witness mentioned character traits such as 'good-looking' or 'he looked like a bank manager' - investigators could perceive this also differently (using rather a piecemeal approach). This process can be applied to most featural descriptors and could be the source of mistakes when constructing the image of the described person. Finally, despite the difficulties in translating a visual image into a verbal description, the police still tend to use person descriptions in their everyday work as one of the primary information-collecting tools (Meissner et al. 2006) or in constructing identification parades that are based on the witness' description of the culprit (McQuiston-Surrett et al. 2006).

\section{Content of person descriptions}

Empirical studies regarding the content of person descriptions began over 30 years ago with an archival study by Kuehn (1974), who analyzed person descriptions provided by victims in 100 police files and found that mostly gender, age, height, build, race, weight, complexion, and hair colour were mentioned (such features were mentioned by more than $70 \%$ of victims, the mean being 7.2 descriptors) but facial features were not often described even though Shepherd (1981) noted that hair, the face outline, the eyes and mouth are important for perceiving and remembering faces and Seitz (2002) found that the eyes and mouth were more important features than the nose in accurate face recognition.

Yuille and Cutshall (1986) examined police files regarding a single shooting incident and found 392 action, 180 person description, and 78 object description 
details (of which $82 \%, 76 \%$, and $89 \%$ turned out to be correct). Most of the errors were for person descriptions regarding height, weight, and age (which have been found hard to describe). There was a lack of memory loss over time, perhaps due to rehearsal, although some specific aspects of the event were forgotten (e.g. some colours, particularly referring to clothing, were not well remembered over time).

Sporer (1992b) in a content analysis of crime files regarding 100 witnesses found that the average number of items provided in the person descriptions was 9.71 and that $22.4 \%$ of the descriptive details referred to general features such as race, age, height, stature, and movements. Another $31 \%$ of the descriptors were about clothes, 29.6\% about the face (mainly hair and beard), 5\% mentioned personality inference, and $12 \%$ 'other' features (e.g. jewellery, dialect, disguise, smell).

Van Koppen and Lochun (1997) found that witnesses in real-life cases mentioned an average of eight descriptors which referred to more general features such as sex, race, and build, rather than features particular to facial characteristics (which have been found harder to describe probably due to lack of vocabulary). The most frequently mentioned characteristics were the gender and height of the perpetrator. Reports of these particular descriptors were completely or partly accurate for more than $80 \%$ of the witnesses and witnesses were fairly accurate in their descriptions of age, build, height, and hair. The descriptions contained more permanent (e.g. gender, race) than temporary characteristics (e.g. clothing, disguises) and overall, of the descriptions, $59 \%$ were correct, $17 \%$ partially correct, and 24\% incorrect. Witnesses were less correct regarding characteristics such as inner face features, dialect, and type of hair. Explanations of this last finding could be that these characteristics are harder to describe due either to a lack of vocabulary or to difficulties in decision-making (i.e. deciding what type of dialect it was). Also, these characteristics can be subjective (i.e. vary across describers) because of variability in how people verbalise them. All these findings support Sporer (1996) who states that it is hard indeed even for adults to translate a rich visual impression into a detailed verbal description whereas the identification of a person at an identification parade, or from a photo spread is an act of visual recognition that is considered to be easier.

Some other characteristics also affect the quality of person descriptions. Time delay between the event and accurately communicating a visual impression to an investigator can influence the quality of testimony. Van Koppen and Lochun (1997) found that witnesses provided fewer person descriptors following longer retention intervals (and that better illumination and shorter distances between the witness and perpetrator were associated with better person descriptions). However, Yuille and Cutshall (1986), found high levels of recall from witnesses of a real crime two years after the incident. Nevertheless, laboratory research has consistently shown significant effects of delay on the accuracy of person descriptions (Ellis et al. 1980, Meissner 2002). 


\section{Characteristics of the targets and witnesses}

\subsection{Height, weight, and age}

As stated above, many witness descriptions contain characteristics such as height, weight, and age of the culprit. However, tall and heavy targets might be underestimated, while short and light targets might be overestimated. This would reflect a general regression toward the mean. Flin and Shepherd (1986) found that observers' own height and weight tended to effect their estimations of the perpetrators' height and weight, but this 'own-anchor' effect was found only for male observers describing a male target. Thus, witnesses' own characteristics might influence their descriptions. Also, the estimations about physical appearance characteristics of perpetrators might also be influenced by witness knowledge about population norms (Meissner et al. 2006).

Manis and Paskewitz (1984) have proposed a judgment model which suggests that prior experience with some members of a given category affects the assessment of other members of the same category in two ways: (i) by providing a basis for comparison and (ii) by leading the judge to expect that new exemplars will resemble those previously encountered. It is generally accepted that estimates of height and weight are relatively inaccurate and thus might be influenced by personal determinants (see Clifford and Bull 1978, Janssen and Horowski 1980).

\subsection{Gender}

It has been found that the gender of a witness can affect descriptions. Women have been found superior to men in recall for targets' weight, hair colour and length (Yarmey 1993), these being attributes that women may have been more likely to attend to at encoding. Powers et al. (1979) found that women were more accurate than men on questions dealing with women's clothing or actions, whereas men were more accurate on questions concerning men's appearance and surroundings. Also, Biernat et al. (1991) found an own-gender effect (people were better at estimating characteristics of persons of their own gender) when participants were estimating target persons' height and weight characteristics.

\section{Empirical research with children}

\subsection{Children's statements and person descriptions}

Even very young children can provide some descriptive details (Sporer 1996). Also, even the youngest of child witnesses are capable of accurately reporting the behaviour of others (Ceci et al. 1998). Regarding verbal recall (which may contain descriptive details), Dekle et al. (1996) found that compared to adults, children's free recall is less complete (but equally accurate). When children are allowed to recall information freely or through the use of general questions, even very young children can produce material that is as accurate as that given by adults (Hutche- 
son et al. 1995). In general, children's testimonies contain significantly fewer details than adults' or adolescents' but the accuracy of the information can be comparable (Davies and Flin 1988, Marin et al. 1979). The fact that adults' statements are usually longer and more detailed than children's (Davies et al. 1989, Dent and Stephenson 1979, Marin et al. 1979) could be due to younger children not encoding and storing information as effectively as adults and older children do (Brainerd et al. 1990). Preschoolers often report relatively little information in response to open-ended invitations for free recall (Poole and White 1991). Prompting children's recall can occasion fuller reporting, but some of this will be inaccurate.

Younger children (nine years old and less) tend to focus on the exterior (e.g. hair) rather than interior (eyes, nose, mouth etc.) facial features of (familiar) others (Davies et al. 1989). Similarly, 10 to 14 year-olds were found to be less accurate than adults in describing interior facial features (e.g. nose), the age, and body characteristics (Pozzulo and Warren 2003). Adults usually describe well the clothing of the perpetrator, children describe the actions, and adolescents describe the appearance (face, body, height, weight) (Cesniene and Bandzeviciene 2005, King and Yuille 1986, cited in Davies 1996). Children's preference for actions could be explained by the notion that when they learn language, they first learn the actions associated with frequently heard verbs (Glenberg and Kaschak 2002). Therefore, they could more notice (and comment on) what a person does than how the person looks. When remembering real-life events, children have been found to be less likely to freely recall descriptive information than central actions and objects (Tobey and Goodman 1992). Pozzulo and Warren (2003) found that adults were better describers both quantitatively and qualitatively than were 10 to 14 year old youths. Adults reported more features of the face and body (height, weigh and build), race, and clothing, whereas youths reported various accessories.

Also, own-group bias has been found in children. For example, Lindholm (2005) found that seven-year-old children had a better recall of own-age than of other-age targets (see also Rehnman 2007).

\subsection{Height, weight, and age}

Although children's accounts are generally accurate (if interviewed properly), their estimates about the person's characteristics (such as height, weight, and age) can be quite poor. Younger children's limited performance with many descriptors could be due to their limited experience of (or exposure to) different persons (see Davies 1996). Davies et al. (1988) found that seven to twelve year old children were poor at estimating the height, weight and age of an unfamiliar person, especially age. Goetze (1980, see in Davies 1996) also found increases with age in the accuracy of estimates of height, weight, and age in 8, 11, and 13-year-old children.

Studies comparing actual and estimated height (Janssen and Horowski 1980) have revealed that the estimations of older children were more correct than of 
younger children. Also, children's own height probably makes it difficult to estimate the height of adult persons. In the case of age estimates, the problem appears to lie in lack of knowledge among younger children of the appropriate facial cues to aging (Ellis 1990) or the concept of aging itself.

To conclude, even young children's accounts can be as accurate as those of adults but not so rich in details. Children tend to focus their attention on different characteristics than adults do. Studies suggest that a developmental trend may be present in that with increasing age, interior features of faces are more noted by children, although they may still be more difficult to describe because they require a richer vocabulary.

\section{Attempts to improve the quality of children's person descriptions}

As mentioned above, children's free recall is accurate but short. To be able to aid children to recall more information about the perpetrator(s), several attempts have been made to improve children's eyewitness testimony but they have had limited success. These have included the usage of anatomical dolls (Ceci and Bruck 1995), a body map (Willcock et al. 2006), a 'Draw and Tell' technique (Poole and Lamb 1998), and using a 'standard model of comparison' (to compare children's memories with a person in their visual field, Kask et al. 2007). As the first three methods are more or less connected to increase the amount of information concerning child abuse cases rather than person descriptions, therefore only the latter method is covered in this review more in depth.

As children's person descriptions and spontaneous recall are usually quite poor, it has been suggested that providing them with possible ranges (to describe the person's height and weight) or a colour plate (to evaluate the correct colour of the person's clothes and hair), may lead to better results for some aspects of person descriptions (Sporer 1996, Meissner et al. 2006). A way that has been suggested to increase the quantity of information children provide about once-seen stranger, is to invite them to provide descriptions relative to a 'standard model of comparison', for example a person in their visual field (Sporer 1996, Meissner et al. 2006). This means that children, when providing descriptions about a once-seen person, can use the person who is interviewing them (e.g. police officer) as a standard when recalling various characteristics of the once-seen person.

However, the provision of a standard did not help adults (Kask et al. 2006) nor six to eight-year-old children (Kask et al. 2007) to recall more information overall about a once-seen person, although the younger children's performance when answering some questions was better when the standard was available. One difficulty is that some children in that study may not have fully understood the idea of trying to use a standard. In conclusion, there have been attempts to aid children's person descriptions but their effect has been minimal. 


\section{Questioning methods with children}

As children's person descriptions are often vague, more information is needed about the person whom the child saw. It is not so much a problem that the child cannot remember, but that the child might not have access to the information in their memory or does not have sufficient verbal skills to actively recall the details about (once-seen) person (Milne and Bull 1999). This section will describe now some issues concerning suggestibility and the influence of different type of questions on children's accounts.

Children are sometimes described as being suggestible (Ceci and Bruck, 1993) and having difficulty distinguishing reality from fantasy (Feher 1988). There are several studies demonstrating that especially preschool children are more suggestible than adults (Ceci et al. 1987, Cohen and Harnick 1980, Ornstein et al. 1992). However, other studies indicate that children are no more suggestible than adults (Marin et al. 1979, Flin et al. 1992). Children's suggestibility is influenced by several factors such as the use of specific or forced-choice questions or repeated questioning (see Crossman et al. 2004 for an overview). Clarke-Stewart, Malloy and Allhusen (2004) found that children who had more advanced verbal abilities, adaptive inhibitory control, close and secure relationships with supportive and psychological healthy parents were better able to resist the interviewer's suggestive questions.

Even very young children are capable of accurate reports if they are questioned in ways that take note of their developmental needs. Because children's free recall can be poor, it is important how police officers interview them. It is common for interviewers to misunderstand children's speech or to overestimate their linguistic capacities (Lamb et al. 1999).

Children's free recall usually contains few information units (Dekle et al. 1996). However, when children are allowed to recall information freely or through the use of general questions, even very young children can produce material which is highly accurate (Hutcheson et al. 1995).

Example of free recall:

What happened then? - I went to park and then he came along. The man pushed me down and didn't let me go.

Example of open-ended question:

Tell me more about the punching? - Umm... It happened all very quickly.

Developmentally it is typically more efficient to ask children general questions, because specific questions or difficult explanations can confuse children and result in increased numbers of false details. Although asking open-ended questions of children could elicit limited information, specific questions can decrease children's overall accuracy which could result in providing an erroneous person description (Hutcheson et al. 1995).

Example of option-posing question:

So you said they forced you to lie down. Was it man \#1 or man \#2 who forced you to lie down? - It was man \#2. 
Example of suggestive question:

Several times a day? How come this happened several times... did it happen then like three times a day? - Yes, three times.

In practice option-posing and suggestive questions are often introduced early in interviews (sometimes as the very first interviewer utterance) and can contaminate subsequent information (Cederborg et al. 2000). Also, there is a tendency in police interviews for interviewers to ask few open questions but many specific and closed questions (Davies et al. 2000, Westcott and Kynan 2006). This could well result in more erroneous statements provided by children. Therefore, it is recommended that interviewers should use as much as possible free recall and open-ended prompts (Home Office 2002) as it is demonstrated that these types of prompts increase the quality and quantity of information compared to other types of questions (Lamb et al. 1996, Sternberg et al. 2001).

However, when children are trained to respond to open-ended prompts in forensic contexts, they produce more information when prompted to "tell everything' (i.e. free recall) about the event or person (Sternberg et al. 1997).

\section{Structured interviewing methods}

The quality of evidence obtained from children is likely to be very dependent on the degree to which police interviewers adhere to best-practice guidelines, as well as the children's general experience with an open-ended style of communication (Agnew et al. 2006). Davies et al.'s (1995) evaluation of field interviews by police in the UK noted that in $43 \%$ of interviews the child was needlessly rushed from the free recall phase to the questioning phase. Even so, we should still ask what would help police officers to collect accurate and detailed person descriptions?

When interviewers use a protocol designed to interview children, they use more recommended practices than those interviewers who do not use the protocol (Orbach et al. 2000). Also, when children are being interviewed using the protocol, they provide more free narrative details than do children interviewed without the protocol.

Therefore, in order to achieve the best balance between quantity and quality in children's accounts, better police training in the adoption of best-practice guidelines in interviewing children is required. It is suggested that police officers be trained to use structured methods such as the NICHD protocol (developed to obtain more information from children especially regarding sexual abuse, see Orbach et al. 2000) or cognitive interview (CI, Fisher and Geiselman 1992). Both of those methods have been found to be effective with children (Milne and Bull 2003) and are therefore strongly recommended.

The basis of using either of those interviewing techniques is to introduce the child to what is going to happen, to decrease the child's level of arousal, and to create a trustful relationship with him/her. This is followed by helping the child to freely recall what happened and then to ask (primarily open-ended) questions and 
apply several mnemonic techniques which could help the child to recall more information.

The amount of information increased $40 \%$ with structured interview methods compared to typical unstructured interviews (Fisher et al. 1989). Also, police officers asked fewer questions in total, more open-ended questions and less suggestive questions, and made more pauses and fewer interruptions. Meta-analysis of Koehnken et al. (1999) revealed a large increase in the number of correct details elicited by the CI. Similar results have been found also for NICHD protocol (Orbach et al. 2000). However, it should be noted that the majority of studies examining the NICHD protocol or the CI have not focused on obtaining person descriptions per se, so further research in this regard would seem worthwhile.

Concerning children, more person, action, and object details were found reported in a CI compared to standard interviewing techniques (Holliday, 2003). Milne and Bull (2003) found that the CI was found to increase the reporting of actions similarly to previous research (Granhag and Spjut 2001) but the accuracy was lower for information about person details (see Memon and Vartoukian, 1996).

Davis, McMahon and Greenwood (2005) studied the effect of MCI (Modified Cognitive Interview, which omitted the change perspectives and change order mnemonics compared to original version of $\mathrm{CI}$ ) on adults and found that $\mathrm{MCI}$ elicited a comparable amount of correct information. The shortened version obtained as much as $87 \%$ of the information of the complete procedure saving approximately $23 \%$ of time. Regarding children, Holliday and Albon (2004) stated that eight-year-olds recalled more correct person, action, object, and location details with MCI than four-year-olds. Therefore, it can be concluded that regarding spontaneous person descriptions by children, the CI and its modifications cannot help to increase the number of details but in terms of questioning the number of details referring to persons or actions can be increased.

When intensive training in the use of a highly structured interview protocol followed by continuing supervision was provided to police officers (as monthly day-long seminars and feedback on all field interviews), then these activities yielded in improvements in interview quality (Lamb et al. 2002). Therefore, this would be a good example how scientific knowledge is integrated into practice (in order to enhance the quality of police interviews with children).

\section{Conclusion}

Various issues concerning children's person descriptions were discussed in this paper. As children's spontaneous person descriptions are short (but accurate), quantitatively and qualitatively more information could be obtained by developmentally appropriate interviewing techniques. Investigators should follow a structured interview protocol and allow children to freely recall information about a person before applying open-ended (and closed) questions. 


\title{
Acknowledgements
}

We are grateful to the two anonymous reviewers for their comments.

\author{
Address: \\ Kristjan Kask \\ University Nord \\ Siili 14 \\ 13413 Tallinn, Estonia \\ Tel.: +372673 7962 \\ E-mail: kristjan.kask@nord.ee
}

\section{References}

Agnew, S. E., M. B. Powell, and P. C. Snow (2006) "An examination of the questioning styles of police officers and caregivers when interviewing children with intellectual disabilities". Legal and Criminological Psychology 11, 35-53.

Biernat, M., M. Manis, and T. E. Nelson (1991) "Stereotypes and standards of judgment". Journal of Personality and Social Psychology 66, 199-214.

Brainerd, C. J., V. F. Reyna, M. L. Howe, and J. Kingma (1990) "The development of forgetting and reminiscence". Monographs of the Society for Research in Child Development 55, v-93.

Breznitz, Z. (2002) "Asynchrony of visual-orthographic and auditory-phonological word recognition processes: an underlying factor in dyslexia". Reading and Writing: An Interdisciplinary Journal 15, 15-42.

Ceci, S. J. and M. Bruck (1993) "Suggestibility of the child witness: a historical review and synthesis". Psychological Bulletin 113, 403-439.

Ceci, S. J. and M. Bruck (1995) Jeopardy in the courtroom. Washington, DC: American Psychological Association.

Ceci, S. J., D. F. Ross, and M. P. Toglia (1987) "Suggestibility of children's memory: Psycholegal implications". Journal of Experimental Psychology: General 27, 38-51.

Ceci, S. J., A. M. Crossman, L. L. Gilstrap, and M. H. Scullin (1998) "Social and cognitive factors in children's testimony". In Eyewitness memory: theoretical and applied perspectives, 15-30. C. P. Thompson, D. J. Herrmann, J. D. Read, D. Bruce, D. G. Payne, and M. P. Toglia, eds. New Jersey: Lawrence Erlbaum Associates.

Cederborg, A.-C., Y. Orbach, and K. J. Sternberg (2000) "Investigative interviews of child witnesses in Sweden". Child Abuse and Neglect 24, 1355-1361.

Cesniene, I. and R. Bandzeviciene (2005) "Gender differences in accuracy of preschool children memory for eyewitnessed event". Paper presented in XVth European Conference on Psychology and Law. July, Vilnius.

Clarke-Stewart, K. A., L. C. Malloy, and V. D. Allhusen (2004) "Verbal ability, self-control, and close relationships with parents protect children against misleading suggestions". Applied Cognitive Psychology 18, 1037-1058.

Clifford, B. R. and R. Bull (1978) “The psychology of person identification”. London: Routledge and Kegan Paul.

Cohen, R., and M. Harnick (1980) "The susceptibility of the child witness to suggestion". Law and Human Behavior 4, 201-210.

Crossman, A. M., M. H. Scullin, and L. Melnyk (2004) "Individual and developmental differences in suggestibility". Applied Cognitive Psychology 18, 941-945.

Davies, G. M. (1996) "Children's identification evidence". In Psychological issues in eyewitness identification, 233-258. S. L. Sporer, R. S. Malpass, and G. Koehnken, eds. New Yersey: Lawrence Erlbaum Associates 
Davies, G. M. and R. Flin (1988) "The accuracy and suggestibility of child witnesses". Issues in Criminological and Legal Psychology 13, 21-34.

Davies, G. M., Y. Stevenson-Robb, and R. Flin (1988) "Tales out of school: children's memory for an unexpected event". In Practical aspects of memory: current research and issues. Vol .1: Memory in everyday life, 122-127. M. M. Gruneberg and P. E. Morris, eds. Oxford: John Wiley \& Sons.

Davies, G. M., A. Tarrant, and R. Flin (1989) “Close encounters of the witness kind: children's memory for a stimulated health inspection". British Journal of Psychology 80, 415-429.

Davies, G. M., H. L. Westcott, and N. Horan (2000) "The impact of questioning style on the content of investigative interviews with suspected child sexual abuse victims". Psychology, Crime \& Law 6, 81-97.

Davies, G. M., C. Wilson, R. Mitchell, and J. Milsom (1995) Videotaping children's evidence: an evaluation. London: Home Office.

Davis, M. R., M. McMahon, and K. M. Greenwood (2005) "The efficacy of mnemonic components of the cognitive interview: Towards a shortened variant for time-critical investigations". Applied Cognitive Psychology 19, 75-93.

Dekle, D. J., C. R. Beal, R. Elliott, and D. Huneycutt (1996) "Children as witnesses: a comparison of lineup versus showup identification methods". Applied Cognitive Psychology 10, 1-12.

Dent, H. and G. Stephenson (1979) "An experimental study of the effectiveness of different techniques of questioning child witness". British Journal of Social and Clinical Psychology $18,41-51$.

Ellis, H. D. (1990) "Developmental trends in face recognition". The Psychologist 3, 114-119.

Ellis, H. D., J. W. Shepherd, and G. M. Davies (1980) "The deterioration of verbal descriptions of faces over different delay intervals". Journal of Police Science \& Administration 8, 101-106.

Feher, T. (1988) "The alleged molestation victim, the rules of evidence, and the Constitution: Should children really be seen and not heard?" American Journal of Criminal Law 14, 227.

Flin, R., J. Boon, R. Bull, and A. Knox (1992) "The effect of a five-month delay on children's and adults' eyewitness memory". British Journal of Psychology 83, 323-337.

Fisher, R. P. and R. E. Geiselman (1992) Memory enhancing techniques for investigative interviewing: the cognitive interview. Springfield, Illinois: Charles C. Thomas.

Fisher, R. P., R. E. Geiselman, and M. Amador (1989) "Field test of the cognitive interview: enhancing the recollection of actual victims and witnesses of crime". Journal of Applied Psychology $74,722-727$.

Flin, R. H. and J. W. Shepherd (1986) 'Tell stories: eyewitnesses' ability to estimate height and weight characteristics". Human Learning 5, 29-38.

Glenberg, A. M. and M. P. Kaschak (2002) "Grounding language in action". Psychonomic Bulletin \& Review $9,558-565$.

Goetze, H. J. (1980) The effect of age and method of interview on the accuracy and completeness of eyewitness accounts. Unpublished doctoral dissertation, Hofstra University, Hempstead, NY.

Granhag, P. A., and E. Spjut (2001) "Childrens's recall of the unfortunate fakir: a further test of the Enhanced Cognitive Interview". In Psychology in the courts: International advances in knowledge, 209-222. R., Roesch, R. R., Corrado, and R. J. Dempster, eds. London: Routledge.

Holliday, R. E. (2003) "Reducing misinformation effects in children with cognitive interviews: Dissociating recollection and familiarity". Child Development 74, 728-751.

Holliday, R. E. and A. J. Albon (2004) "Minimising misinformation effects in young children with cognitive interview mnemonics". Applied Cognitive Psychology 18, 263-281.

Home Office (2002). Achieving best evidence in criminal proceedings: guidance for vulnerable or intimidated witnesses, including children. London: Home Office Communication Directorate.

Hutcheson, G. D., J. S. Baxter, K. Telfer, and D. Warden (1995) "Child witness statement quality: question type and errors of omission". Law and Human Behavior 19, 631-648.

Janssen, J. P. and A. C. Horowski (1980) "Students' estimates of stature: tendency to accentuate as a cognitive style in person perception". Zeitschrift für Entwicklungspsychologie und Pädagogische Psychologie 12, 167-176. 
Kask, K., R. Bull, and G. M. Davies (2006) "Trying to improve young adults' person descriptions". Psychiatry, Psychology, and Law 13, 174-181.

Kask, K., R. Bull, I. Heinla, and G. M. Davies (2007) "The effect of a standard to improve person descriptions by children". Journal of Police and Criminological Psychology 22, 77-83.

King, M. A. and J. C. Yuille (1986) An investigation of the witness abilities of children. Unpublished manuscript, University of British Columbia.

Koehnken, G., R. Milne, A. Memon and R. Bull (1999) "The cognitive interview: a meta-analysis". Psychology, Crime and Law 5, 3-28.

Koppen, van P. J. and S. K. Lochun (1997) "Portraying perpetrators: the validity of offender descriptions by witnesses". Law and Human Behavior 21, 661-685.

Kuehn, L. L. (1974) "Looking down a gun barrel: person perception and violent crime". Perceptual and Motor Skills 39, 1159-1164.

Lamb, M. E., I. Hershkowitz, K. J. Sternberg, B. Boat, and M. D. Everson (1996) "Investigative interviews of alleged sexual abuse victims with and without anatomic dolls". Child Abuse and Neglect 20, 1239-1247.

Lamb, M. E., K. J. Sternberg, Y. Orbach, I. Hershkowitz, and P. W. Esplin (1999) "Forensic interviews of children". In The psychology of interviewing: a handbook, 253-277. R. Bull and A. Memon, eds. Chicester: Wiley.

Lamb, M. E., K. J. Sternberg, Y. Orbach, I. Hershkowitz, D. Horowitz, and P.W. Esplin (2002) "The effects of intensive training and ongoing supervision on the quality of investigative interviews with alleged sex abuse victims". Applied Developmental Science 6, 114-125.

Lindholm, T. (2005) "Own-age biases in verbal person memory". Memory 13, 21-30.

Manis, M. and J. Paskewitz (1984) "Judging psychopathology: expectation and contrast". Journal of Personality and Social Psychology 20, 363-381.

Marin, B. V., D. L. Holmes, M. Guth, and P. Kovac (1979) "The potential of children as eyewitnesses". Law and Human Behavior 3, 295-305.

McQuiston-Surrett, D., R. S. Malpass, and C. G. Tredoux (2006) "Sequential vs. simultaneous lineups: a review of methods, data, and theory". Psychology, Public Policy, and Law 12, $137-169$

Meissner, C. A. (2002) "Applied aspects of the instructional bias effect in verbal overshadowing". Applied Cognitive Psychology 16, 911-928.

Meissner, C. A., S. L. Sporer, and J. F. Schooler (2006) "Person descriptions as eyewitness Evidence". In Handbook of eyewitness psychology: memory for people, 3-34. R. C. L. Lindsay, D. F. Ross, J. D. Read, and M. P. Toglia, eds. Mahwah, NJ: Lawrence Erlbaum.

Memon, A., and R. Vartoukian (1996) "The effects of repeated questioning on young children's eyewitness testimony". British Journal of Psychology 87, 403-416.

Milne, R. and R. Bull (1999) Investigative interviewing: psychology and practice. Chichester: John Wiley \& Sons.

Milne, R. and R. Bull (2003) "Does the cognitive interview help children to resist the effects of suggestive questioning?" Legal and Criminological Psychology 8, 21-38.

Orbach, Y., I. Hershkowitz, M. E. Lamb, K. J. Sternberg, P. W. Esplin, and D. Horowitz (2000) "Assessing the value of structured protocols for forensic interviews of alleged abuse victims". Child Abuse and Neglect 24, 733-752.

Ornstein, P. A., B. N. Gordon, and D. M. Larus (1992) "Children's memory for a personally experienced event: Implications for testimony”. Applied Cognitive Psychology 6, 49-60.

Paivio, A (1969) "Mental imagery in associative learning and memory". Psychological Review 76, 241-263.

Paivio, A. (1971) Imagery and verbal processes. New York: Holt, Rinehart \& Winston.

Poole, D. A. and M. E. Lamb (1998) Investigative interviews of children: a guide for helping professionals. Washington, DC: APA.

Poole, D. A. and L. T. White (1991) "Effects of question repetition on the eyewitness testimony of children and adults". Developmental Psychology 27, 975-986.

Powers, P. A., J. L. Andriks, and E. F. Loftus (1979) "The eyewitness accounts of females and males". Journal of Applied Psychology 64, 339-347 
Pozzulo, J. D. and K. L. Warren (2003) "Descriptions and identifications of strangers by youth and adult eyewitnesses". Journal of Applied Psychology 88, 315-323.

Pozzulo, J. D., J. M. T. Lemieux, E. Wells, and H. J. McCuaig (2007) "The influence of eyewitness identification decisions and age of witness on jurors' verdicts and perceptions of reliability". Psychology, Crime, \& Law 12, 641-652.

Rehnman, J. (2007) The role of gender in face recognition. Doctoral dissertation: Stockholm University.

Schepard, A. I. (2004) Children, courts, and custody: interdisciplinary models for divorcing families. New York, US: Cambridge University Press.

Seitz, K. (2002) "Parts and wholes in person recognition: Developmental trends". Journal of Experimental Child Psychology 82, 367-381.

Shepherd, J. W. (1981) "Social factors in face recognition". In Perceiving and remembering faces, 55-79. G. Davies, H. Ellis, and J. Shepherd, eds. London: Academic Press.

Shepherd, J. W. and H. D. Ellis (1996) "Face recall - methods and problems". In Psychological issues in eyewitness identification, 87-116. S. L. Sporer, R. S. Malpass, and G. Koehnken, eds. Mahwah, New Yersey: Lawrence Erlbaum Associates.

Sporer, S. L. (1992a) "Post-dicting eyewitness accuracy: confidence, decision-times and person descriptions of choosers and non-choosers". European Journal of Social Psychology 22, 157-180.

Sporer, S. L. (1992b, March) "An archival analysis of person descriptions". Paper presented at the Biennial Meeting of the American Psychology-Law Society in San Diego, California.

Sporer, S. L. (1996) "Psychological aspects of person descriptions". In Psychological issues in eyewitness identification, 53-86. S. L. Sporer, R. S. Malpass, and G. Koehnken, eds. New Jersey: Lawrence Erlbaum Associates

Sternberg, K. J., M. E. Lamb, G. M. Davies, and H. L. Westcott (2001) "The memorandum of good practice: theory versus application". Child Abuse \& Neglect 25, 669-681.

Sternberg, K. J., M. E. Lamb, I. Hershkowitz, L. Yudilevitch, Y. Orbach, P. W. Esplin, and M. Hovev, M. (1997) "Effects of introductory style on children's abilities to describe experiences of sexual abuse". Child Abuse \& Neglect 21, 1133-1146.

Tobey, A. E. and G. S. Goodman (1992) "Children's eyewitness memory: effects of participation and forensic context." Child Abuse \& Neglect 16, 779-796.

Wegner, M. J. and E. M. Ingvalson (2002) "A decisional component of holistic encoding”. Journal of Experimental Psychology: Learning, Memory, and Cognition 28, 872-892.

Westcott, H. L. and S. Kynan (2006) "Interviewer practice in investigative interviews for suspected child sexual abuse". Psychology, Crime \& Law 12, 367-382.

Willcock, E., K. Morgan, and H. Hayne (2006) "Body maps do not facilitate children's reports of touch". Applied Cognitive Psychology 20, 607-615.

Yarmey, A. D. (1993) "Adult age and gender differences in eyewitness recall in field settings". Journal of Applied Social Psychology 23, 1921-1932.

Yuille, J. C. and J. L. Cutshall (1986) "A case study of eyewitness memory of a crime". Journal of Applied Psychology 71, 291-301. 